\title{
The Linguistic Perspectives on Computer Mediated Communication
}

\author{
Larry Dwan Chong \\ larrychong@hotmail.com \\ School of Foreign Languages \& Tourism \\ Gyeongju University \\ Gyeongju, Republic of Korea
}

\begin{abstract}
This paper aims to explore the role of production and perception constraints in computer mediated communication. I review Lindblom's (1990) theory of phonetic variation and propose a new model of linguistic production in Computer Mediated Communication. Cyber citizens use cyber communication as conceptually oral, medially written. The reason to use chat-mode is that it saves time and space (the principle of least effort); here sound, not spelling, is the first thing to be considered. With respect to production in the proposed model, effort is no longer equated with articulatory movement, but rather with the number of keystrokes involved in typing an utterance. On discussing online, discussants show paralinguistic actions such as smile, frown, screaming, etc., and they also reduplicate writings, capitalize all the sentences, and use emoticons; net-communication is headed toward less grammatical and more telegraphic type. The production of hyper-and hypo-forms such as reduplication, punctuation and capitalization will vary according to the sender's estimation of signal-complementary processes and his attempts to compensate for the restricted context. We discuss online and off line on the issues; why we like cyber communication and how we classify the phenomena. The more computer mediated communications we use, the more issues we have to review beyond words and linguistic principles.
\end{abstract}

Keywords: computer-mediated communication, emoticon, acronym, linguistic constraints

\section{Introduction}

All over the world, the growing popularity of the internet (web) and the increasing familiarity of Computer Mediated Communication (CMC) are new social phenomena. This internet technology is, however, often seen as a source of separation between human beings as an obstacle in interpersonal communication. Internet technologies have given rise to multiple forms of interactive written discourse. Synchronous communication systems, such as Internet Relay Chat (IRC), allow users to have real-time conversations that combine characteristics of face-to-face verbal interaction with those of text-based discourse.

Communication in IRC consists of typewritten exchanges that take place in real-time among users who are spatially distant. These exchanges are transmitted, received and responded to within a time frame formerly thought relevant only to spoken communication. With respect to the IRC user community, Reid (1991) notes that members are preselected by external social structures and tend to occupy an economically privileged position in their society. Users tend to belong to the

\footnotetext{
${ }^{1}$ This article is accepted and will be published in Beyond Words Vol.8 No.1 May 2020.
}

DOI: https://doi.org/10.33508/bw.v8i1.2366 
academic and research community and have access to computer technology and the internet. Since they have no prior knowledge of each other, users interact anonymously in the knowledge that they are in rough equality.

Studies of the linguistic features of Computer Mediated Communication suggest similarities with processes that occur in real world communication. For example, Werry (1996) notes the high frequency of reduction strategies that function to reduce the time and effort necessary to communicate in terms like "orthographic reduction and omission of pronouns, etc. also resembles phonological reduction and ellipsis in rapid, informal speech, rendering chat exchanges 'speech-like' in their degree of informality as well" (1996, p.56).

This tendency toward reduction has a parallel in contemporary phonological and morphological theory. This explanation is based on the notion that speech production involves reconciling two conflicting constraints: minimization of articulatory effort and maximization of perceptual discrimination (Linblom, 1990; Mohanan, 1993; Byrd, 1994). These accounts continue a line of research on phonetic explanation in phonology, whereby phonological patterns are seen as the result of articulatory or perceptual factors. The parallel with Internet Written Discourse (IWD) in CMC raises an interesting question: to what extent can patterns of linguistic reduction in IWD be explained in terms of production and perception?

In this paper, we explore the role of production and perception constraints in the IWD register of English. In particular, we address the following questions; what modules govern reduction processes in real world speech? What modules govern reduction processes in IWD?; How do English IWD users modify linguistic forms in accordance with these modules?

This paper first reviews Lindblom's (1990) Hypo \&Hyper (H \& H) Theory of phonetic variation in speech production. Second, Computer Mediated Communic-ation and Hypo- and Hyper-form of cyber communication is introduced and simply the $\mathrm{H} \& \mathrm{H}$ theory to CMC production is applied to review the similarities. We open a stage to discuss it on and off line among CMC researchers and propose a new model of linguistic production in CMC. We conclude with some remarks and implications.

\section{Accounting for Linguistic Variation in Real Speech}

A fundamental problem in phonetic theory is that of invariance. Because speech sounds undergo modification in context, a linguistic category cannot be given a constant phonetic definition. Lindblom (1990) proposed a theory to explain phonetic variation in speech production. According to the $\mathrm{H} \& \mathrm{H}$ theory, speech production is adaptive and involves an inter-play between production- oriented factors and output-oriented constraints. Speakers tune their performance according to communicative and situational demands.
Phonetic output is expected to vary along a continuum from hypo-speech, or reduced forms, to hyper-speech, or non-reduced forms. More recently, these production and perception factors have resurfaced as formalized constraints in Jun's (1995) Optimality Theoretic account of gradient assimilation.

This hypothesis reflects Lindblom's (1990) characterization of speech production as the reconciliation of conflicting production and perceptual factors. Figure 1 gives Lindblom's visual 
representation of the $\mathrm{H} \& \mathrm{H}$ theory of phonetic variation.

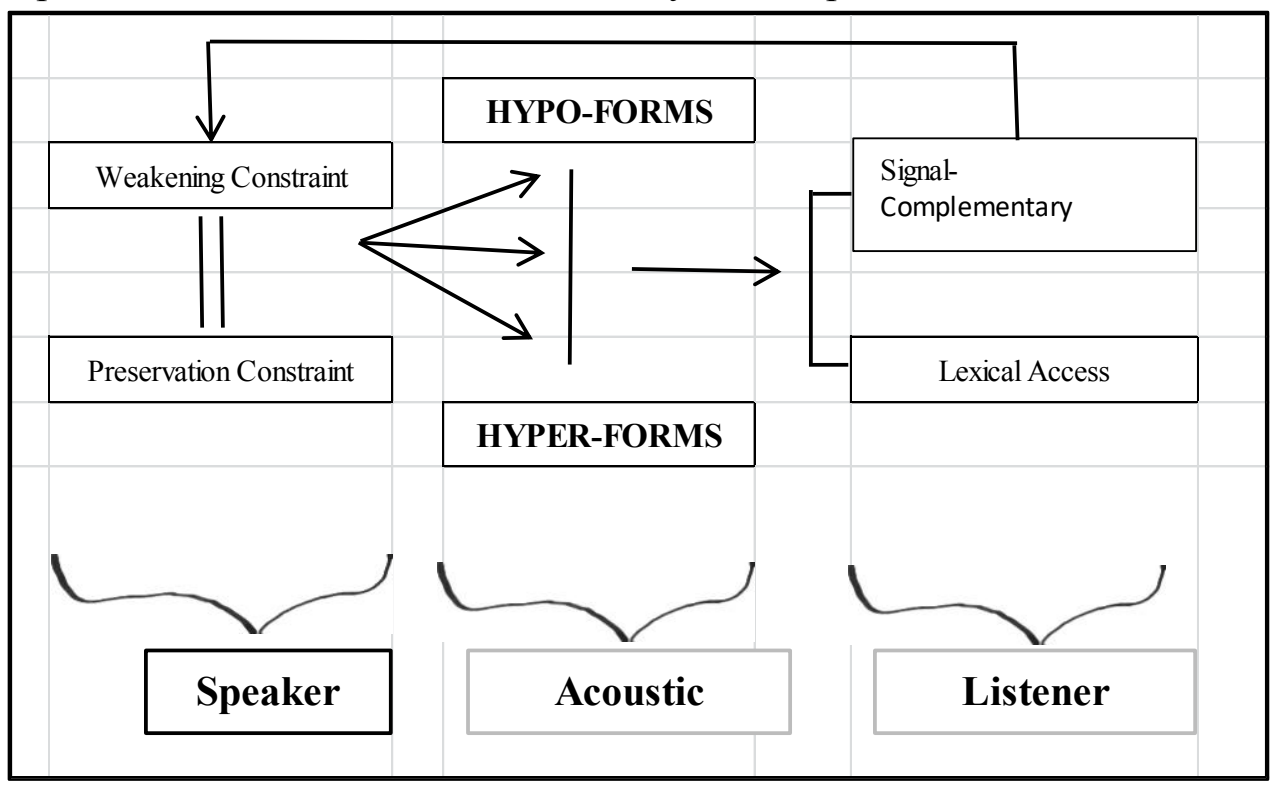

Fig. 1. A model of phonetic variation in speech production

According to the $\mathrm{H} \& \mathrm{H}$ theory, speech perception involves discrimination among items stored in the listener's lexicon. Lexical access is a function of the distinctiveness (rather than invariance) of the acoustic stimulus. Access is facilitated by knowledge that is not present in the signal, i.e., signalcomplementary process. With respect to speech production, Preservation Constraints tend to yield hyper-speech forms, which ensure perceptibility of the acoustic stimulus. As output constraints diminish, the Weakening Constraints tend to yield hypo-speech forms, which involve minimal articulatory effort. In producing an utterance,

\section{The Understanding of CMC Language}

\section{CMC Language}

Considering CMC language, the sender or receiver can choose to have a printed page version, but there is no requirement to print it in order to read it. Some linguists have recognized the fact that Computer Mediated Communication is a hybrid register that resembles both speech and writing and yet is neither, distinguishing 'web speech' as a completely unique mode of communication over the electronic the speaker estimates the running contribution that signal-complementary processes will make on the listener's side and varies his production accordingly along a continuum of hypo-and hyper-speech forms. The difference between hypo- and hyper-forms is illustrated in examples (1):

(1) a. I am not going to wash the car today.

b. 'm nahgonna wash 'duh car d'day. The utterance in (1a) is situated at the hyper end of continuum since it involves preservation of segmental content, whereas that in (1b) is a hypo-form involving articulatory reduction.

medium. Here we can combine the definition of $\mathrm{CMC}$ language as a language which is used to communicate in the net-mediated world.

Perhaps we could even trace back the origin of these jargon words, abbreviations, or phonological simplifications. In the beginning of the 19th century, Morse-code jargon used by radio amateurs and either of two codes consisting of variously spaced dots and dashes or long and short sounds 
used for transmitting messages by audible or visual signals. In the 1930's, Telex ( blended from teleprinter + exchange) began to replace the Morse-code system and this communication service involves teletype machines connected by wire. As the amount of communication has grown in modern society, the Facsimile (L. facere, make + simile, like) was developed to transmit and reproduce even graphic matter by means of signals sent through telephone lines in the early 1960's. The up-to-date computer system allows us to communicate by means of immediate and stored sending and receiving messages electronically between terminals linked by telephone lines of microwave relays, or hard-wired systems such as local access networks (LAN). Applying the proposed model of real speech to Computer-mediated communication, the communication will be between sender and receiver instead of speaker and listener. Perception involves parsing the alphabetical string into distinctive corresponding lexical items. With respect to production, effort is no longer equated with articulatory movement, but rather with the number of keystrokes involved in typing an utterance. Temporal Constraints imposed by IWD favor minimal effort strategies in order to reduce the time and effort necessary to communicate.

\section{Hypo- and Hyper-forms in CMC}

The utterances presented in this section are taken from study on CMC by Werry (1996). The examples in (2)-(5) illustrate hypo-forms, which may be understood as the result of minimal effort strategies employed by the sender as a response to temporal constraints.

(2) Deletion/reduction of subject pronouns

a. $<$ Larry $>$ t pardonee Diva. 8 -) b. <John> goodby gonna try and do something smart for once.

c. $<$ Kath $>$ in a bad mood:(

(3) Abbreviation / truncation

a. $<$ Hari $>$ can you get rid of the auto kick pls?

b. $<$ Bomber $>$ where $\mathrm{r} u$ from?

c. $<$ bubu $>$ well i gotta go ...cu

(4) Acronyms
a. atm at the moment
b. brb be right back
c. convo conversation
d. filfre feel free
e. irl in real life
f. lol laugh out loud

(5) Emoticons
a. :) a smiling face
b. :( an unhappy face
c. 8-) someone wearing glasses
d. :-o someone shocked
e. :-X someone's lips sealed
f. :-Q someone smoking
g. ;-) someone winking
h. :-@ someone screaming

The forms in (2) involves syntactic reduction, while those in (3)-(4) show orthographic simplification in comparison to their standard forms. The emoticons in (5) represent a symbolic type of reduction in that they do not correspond to an explicit canonical form as do the hypo-forms in (2)-(4). Nonetheless, there must be some agreed-upon meaning that IRC users ascribe to them. The emoticon in (5a), for example, can serve as shorthand for a number of meanings, depending on the context of use: "Do not be offended," "I'm just kidding," "I'm happy about what you just said," etc.

The utterances in (6)-(8) contain instances of hyper-forms and are thus situated at the opposite end of the reduction continuum.

(6) Reduplication to indicate drawn-out or expressive intonation 
a. $<$ Lili $>$ Hainan Island? cooolll

b. <Larry $>$ baaaaad joke

lili ...hehehe

c. <Lili $>$ awwwwwwwwww, cool :)

(7) punctuation to create pauses and indicate tempo

cw $7 \mathrm{r}$

a. $<$ Keel $>$ what a peculiar name ...

b. <Larry $>$ hahaha - One of the question of KEEL - where do

most virgins live

c. $<$ Keel $>$ Virginia ... hahahaha

(8) Capitalization reserved for expression of emphasis or shouting a. $<$ Larry $>$ sorry, $\mathrm{m}$ I late too long?

b. $<\mathrm{cw} 7 \mathrm{r}>$ YEEEEES, WAITING FOR YOU TOO LOOOONG

c. <Larry $>$ I cant less than go Wooooooooow

The examples in (6)-(8) show how reduplication, punctuation and capitalization yield hyper-forms, all of which involve greater time and effort (i.e., keystroke combinations) on the part of the sender. They reflect the sender's efforts to compensate for restricted communicative context via textual recreation of non-verbal cues such as intonation, tempo and emphatic speech.

\section{Discussion}

\section{Discussion online}

When I posted the following question via e-mail: why cyber citizens love to use as many acronyms as they could, why it occurs all over the sentences, how we could classify these phenomena, how we can foretell its future, surprisingly, I received more than twenty replies from ten countries. I describe some of discussions.

Why cyber citizens love to use acronyms; we describe chat communication as conceptually oral, medially written. Schrammel (2016) thinks that using acronyms helps to stay within a communication, to hold the attention because of being able to react real quick. She further thinks that acronyms serve a certain expressive function: because of spatio-social constraints (first of all not being able to see each other) there is something new used to convey more than mere information, we can use expressions like 'lol (laugh out loud), rofl (rolling on the floor laughing), etc. as some sort of contextualization. She also thinks that smileys in a certain use serve a similar function: because of not being able to use intonation or mimics to put an utterance how to understand what I said-frame, the chat community creates something new to serve this function.

This phenomenon also occurs in text messages sent by smart (mobile) phones. The primary reason is that it saves time and space. Ruuskanen (2016) states E-mails in Mobile phones are charged by time spent typing msgs (messages) which currently allow only some 150 characters and there are also lots of iconic usages: 'lots' indicates emphases or italics, LOTS indicates shouting, *lots* indicates emphasis or irony, and so on. These are due to the fact that e-mail does not allow italics, etc. and originally only allowed capital letters. The 'smileys' like :-) indicate emotion, sarcasm, irony, or 'don't take me seriously'-it is very hard to indicate emotion in writing when you are limited as to time and space.

Anipa (2016) believe the abundant use of acronyms in cyber communication is very natural, exemplifying what Ferdinand de Saussure (1916) called the principle of least effort (later dubbed 'Language Economy', etc.). She thinks humans subconsciously try 
to find the most economical way of putting their messages across to the interlocutor. This linguistic behavior is reinforced by the

speedy nature of human life in general, particularly in the 20th century. She insists again the written form of language is the artificial way of representing the natural phenomenon of language; and, obviously, being artificial, it is far from being perfect, hence writing is sometimes felt to be an impediment to natural communication.

Lotfi (2016) says that the first thing that comes to the mind is that economy must be behind it. But as usual the first impression may not be the answer. Most probably the phenomenon does have its roots in economy considerations telegram - telex - and e-mail users had in the past in order to save the time needed for sending a message and as a result to reduce the costs. But e-mail users today must have different reasons for that because charges are not that high any more. A few minutes ago, an e-mail arrived urging him to answer ASAP (as soon as possible). In his reply, he mentioned 'I couldn't answer ASAPer than this!' Interestingly enough, the sender of that e-mail was one of those 'busy people' who have no time to even capitalize their sentences. His impression is that e-mail has become something like 'fast food' (especially pizzas in Iran) for the youth: it has connotations of freedom, informality, a rebel against the fixed traditions of the business, and an attempt to avoid clichés. It's even similar to the function of slangy expressions in communications of freshness and novelty.

McHale (2016) claims that we use acronyms, speed writing (pls) and emoticons as a way of compensating for the lack of body English, intonation and other phenomena that we use in conversation to more clearly make out point. Cyber citizens view e-mail as both informal and transient therefore it's $\mathrm{OK}$ to add emoticons to the mix. He thinks that often that view is wrong. Certainly e-mail isn't short-lived as the White House e-mail, the Microsoft anti-trust case and numerous other legal cases have shown.

Zhang (2016) claims that they love to use some acronyms such as BTW, but not as many as they could, because those demand too much effort from the interlocutors when their English proficiency is low. It seems to her that acronyms may trigger the imagination of the netizens. She thinks that here the language use is a game. When such acronyms are used often in talking, it is the principle of economy that plays a role here, and/or, conditioned reaction to the stimulus (message to be conveyed in the required phonological form). In such a language behavior, sound comes first. The written (typed) words only serve the purpose of triggering the phonological representation of the concepts.

How we could classify these phenomena; Even in real language, it is not easy to classify acronyms. Joosten (2016) thinks there is a substantial difference between BTW ( $<$ by the way) and R U ( $<$ are you). BTW is an abbreviation, R U is not, he thinks, or certainly not a normal one. What happens with $\mathrm{R} \mathrm{U}$ is that sounds are transmitted to a new graphic form, a new spelling form (other examples are XTC for 'ecstasy', U2 for 'you too', INXS for 'in excess'). Once acronyms are created (e.g., CIA, GATT), the sound of the original base-form is of no importance: its letters that are selected and brought together not sound. For instance, CIA pronounces as 'see-ai-ei', the ai-sound is not identical to the i-sound in 'intelligence' (Central Intelligence Agency).

Viredaz (2016) states he would not use the word 'acronym' for e.g. BTW, because 
such an abbreviation is not noun, i.e., it is not syntactically used as a noun. In any case, the phenomenon involves all sort of abbreviations, be they proper acronyms

(NLP for Natural Language Processing), other abbreviations using initials (IMO), rebus (how R U), simplified spellings (such as hi->high, lo->low, though these are not restricted to the Net).

Lisecki (2016) refers to the whole phenomenon as an 'Abbreviated - talk mode', simply saying it's a feature supported on any Unix (and some other systems) that allows two or more logged-in users to set up a real-time chat. He guesses that one could even trace back the origin of these gargon (jargon) words, abbreviations, or phonological simplifications, to the times of the Morse-code jargon used by radio amateurs at the beginning of the century.

How we can foretell its future; Barbara (2016) replies what we find really interesting in the topic is the question how far you can go with creating new acronyms and smileys and whether they get acknowledged by the chat community. Where is net talk headed? McHale (2016) states we are not sure today, but we would guess the trend is toward less syntax (pro drop, phrases instead of sentences) and more telegraphic type speech.

Summarizing discussion on line; cyber citizens think chat communication as conceptually oral, medially written: the reason to use chat-mode is that it saves time and space (the principle of least effort): sound, not letter is the first thing to be considered: to express paralinguistic actions such as smile, frown, screaming, etc., they duplicate writings, capitalize all the sentences, and use emoticons: net-communication is headed toward less syntax and more telegraphic type.

\section{Discussion off line}

One interesting point in $\mathrm{CMC}$ is that users do not just reduce written forms all the time instead, when necessary, they duplicate writings, capitalize all the sentences, and use emoticons. This means the principle of economy alone cannot fully explain the phenomenon on line they use.

In computer-mediated communication, the communication will be between sender and receiver instead of speaker and listener. Perception involves parsing the alphabetical string into distinctive corresponding lexical items. With respect to production, effort is no longer equated with articulatory movement, but rather with the number of keystrokes involved in typing an utterance. We have seen temporal constraints imposed by IWD favor minimal effort strategies in order to reduce the time and effort necessary to communicate.
It is claimed that we may explain the phenomenon as we take into account two additional factors not present in Lindblom's $\mathrm{H}$ \& $\mathrm{H}$ model. These factors reflect additional constraints on production and perception that are imposed by the Internet medium. First, Werry (1996) notes the rapidity with which communicative exchanges take place on IRC. Typed utterances appear on screen in chronological orders received by the IRC system, making impossible the overlaps and interruptions of normal face-to-face verbal communication. Disparate strands of conversation are juxtaposed, creating an ongoing and multidimensional text that scrolls up and off each person's screen at a fast pace.

Second, Reid (1991) argues that communication on IRC takes place in a restricted context. In face-to face interaction, non-verbal information (smiles, frowns, 
tones of voice, posture, etc.) provides a context for interlocutor contributions. Since IRC is a text-based medium, the non-verbal cues are no longer present. Deprived of speech subtleties and non-verbal cues, utterances no longer express what they are intended to express. Although $\mathrm{CMC}$ is synchronous like face to face communication, it imposes additional temporal and contextual constraints that face-to face interaction does not. These constraints are expected to have an impact on reduction processes. Figure 2 presents a model of linguistic reduction which incorporates the additional constraints imposed by the IRC medium.

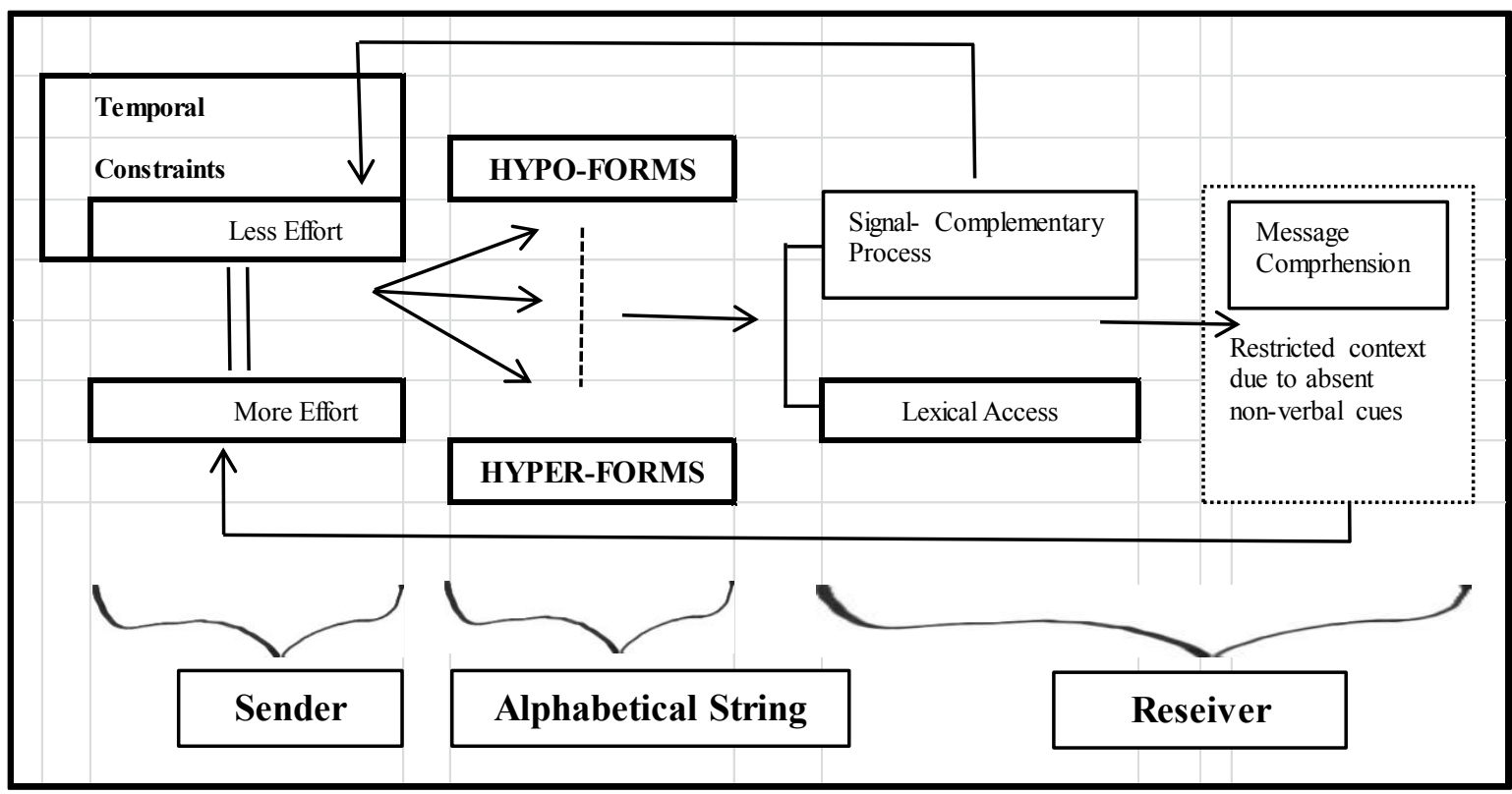

Fig. 2 A proposed model of linguistic production in CMC

According to the proposed model, perception involves parsing the alphabetical string into distinctive corresponding lexical items. As in the $\mathrm{H} \& \mathrm{H}$ model, signal-complementary processes are assumed to facilitate this parsing. With respect to production, effort is no longer equated with articulatory movement, but rather with the number of keystrokes involved in typing an utterance. Temporal Constraints imposed by IWD favor minimal effort strategies in order to reduce the time and effort necessary to communicate.
Additionally, the lack of non-verbal cues restricts the communicative context, creating a potential for miscomprehension by the receiver. The sender must compensate for this restriction by expending more effort to ensure message comprehension. The model predicts that the production of hypo- and hyper-forms will vary according to the sender's estimation of signal-complementary processes and his attempts to compensate for the restricted context.

\section{Conclusion and Suggestion}

The paper has reported that communication in IRC consists of typewritten exchanges that take place in real-time among users who are spatially distant. The high frequency of reduction strategies functions to reduce time and effort necessary to communicate. This tendency toward reduction has parallel in contemporary phonological and morphological theory. 
Based on the notion that speech production involves reconciling two conflicting constraints, we minimize articulatory effort and maximize perceptual discrimination. As compared to Lindblom's $\mathrm{H} \& \mathrm{H}$ model, effort is no longer equated with articulatory movement, but rather with the number of keystrokes involved in typing an utterance.

With respect to linguistic register, Ferguson (1994) states that a communicative situation that recurs regularly in a society will tend over time to develop identifying markers of linguistic structure and language use, different from the language of other communication situations. The samples of hypo- and hyper-forms examined in this paper constitute linguistic markers that identify $\mathrm{CMC}$ as an independent linguistic register. It is by making use of these forms that CMC users are identified as an independent linguistic community.

It is also claimed that CMC users think computer mediated communication as conceptually oral, medially written. The reason to use chat-mode is that it saves time and space (the principle of least effort), but today no longer $\mathrm{CMC}$ users focus time savings, rather it is a kind of linguistic culture. Sound, not letter is the first thing to be considered and they also express paralinguistic actions such as smile, frown, screaming, etc., duplicate writings, capitalize all the sentences, and use emoticons. We could see cyber communication is headed toward less syntax and more telegraphic type.

The occurrence of reduced and exaggerated linguistic forms in CMC has implications for language research and acquisition as technology continues to be integrated into foreign language courses. The use of synchronous communication systems in this context will potentially expose L2 learners to input that varies along the hypo \& hyper continuum. This raises several issues that merit further investigation, namely; How do we classify cyber linguistic phenomena in the linguistics? How do cyber languages affect language acquisition? Are there pedagogical advantages or disadvantages in exposure to reduced and exaggerated linguistic forms? How do the temporal and contextual constraints of CMC affect negotiation of meaning and interaction between and among language learners? The more computer mediated communications we use, the more issues we have to review beyond words and linguistic principles.

\section{(C) Larry Dwan Chong}

Larry D. Chong has taught Linguistics, EFL, CALL at Gyeongju University and Western Illinois University over 30 years. As a founder of ASIA CALL, he has given keynote speeches for several International Conferences. He has served as an Editor-in-Chief for the ASIA CALL Journal and as a columnist for the Linguist List.

* I would like to express my great thanks to anonymous reviewers and I am indebted to Prof. Wuri Soedjatmiko for useful comments. 


\section{References}

Byrd, D. 1994. Articulatory Timing in Lindblom, B. 1990. Explaining Phonetic English Consonant Sequences. (Doctoral Dissertation, UCLA). Retrived February 01, 2020, from https://www.amazon.com//ko/gp/produc $\mathrm{t} /$

B0006PA8EM/ref=dbs_a_def_rwt_hsch _vapi_taft_p1_i4/

Cherny, L. 1996. The MUD Register: Conversational Models of Action in a Text-Based Virtual Reality. (Doctoral Dissertation, Standford University). Retrived February 01, 2020, from https://searchworks.stanford.edu/view/3 105436/

Ferguson, C. 1994. Dialect, Register, and Genre: Working Assumptions about Conversationalization, in D. Biber and E. Finegan (eds.) Sociolinguistic Perspectives on Register, 15-30. New York: Oxford University Press.

Jun, Jongho (1995). Place assimilation as the result of conflicting perceptual and articulatory constraints. In Proceeding of WCCFL 14. 221-237. Retrived February 01, 2020, from https://www.researchgate.net/scientificcontributions/2162909855_Jongho_Jun Variation: A Sketch of the $\mathrm{H} \& \mathrm{H}$ Theory, in W. J. Hardcastle and A. Marchal (eds.) Speech Production and Speech Modeling, 403-439. Dordrecht: Kluwer Academic Publishers.

Mohanan, K.P. 1993. Fields of Attraction in Phonology, in J. Goldsmith (ed.) The Last Phonological Rule: Reflections on Constraints and Derivations, 61-116. Chicago: The University of Chicago Press.

Reid, E. 1991. Electropolis: Communication and Community on Internet Relay Chat. (Senior Honours thesis, University of Melbourne, Australia). Retrived February 01, 2020, from http://www.aluluei.com/electropolis.ht $\mathrm{m} /$

Werry, C. 1996. Linguistic and Interactional Features of Internet Relay Chat. In Susan Herring (ed.) Computer Mediated Communication: Linguistic, Social and Cross-Cultural Perspectives, 47-64. Amsterdam: John Benjamins Publishing Company.

\section{Personal Communications}

Ahmad Reza Lotfi, English Department, Azad University, Iran.

Barbara Schrammel, Department of Linguistics Graz, Austria.

Deborah D. Kela Ruuskanen, Leankuja, Vantaa Finland.

Frank Joosten, Department of Linguistics, K. U. Leuven, Belgium.

Kormi Anipa, School of Modern Languages, University of St. Andrews, United Kingdom.

Michal Lisecki, Institute of Slavonic Studies, University of Silesia, Poland.

Michael McHale, MNIS TextWise Labs, USA.

Remy Viredaz, Geneva, Switzerland.

Wenzhong Zhang, Foreign Languages School, Wuhan University, China. 
Appendix 1. Acronyms

\begin{tabular}{ll}
\hline Acronym & Meaning \\
\hline ATM & at the moment \\
\hline ASAP & as soon as possible \\
\hline BRB & be right back \\
\hline BTW & by the way \\
\hline CU L8R & see you later \\
\hline FWIW & for what it's worth \\
\hline FYI & for your information \\
\hline HTH & hope this helps \\
\hline IMHO & in my humble opinion \\
\hline IMO & in my opinion \\
\hline IOW & in other words \\
\hline IRL & in real life \\
\hline LOL & laugh out loud \\
\hline OIC & oh, i see \\
\hline ROTFL & rolling on the floor laughing \\
\hline
\end{tabular}

Appendix 2. Emoticons : E-Mail Body Language

\begin{tabular}{|c|c|c|}
\hline Face & Interpretation & Alternative interpretation \\
\hline :-) & Ha ha smile & This is supposed to make you laugh \\
\hline$(-:$ & Submitter is left-handed & \\
\hline |-) & Hee hee & Submitter is asleep (out of boredom) \\
\hline |-D & Но Но & \\
\hline$:-\mathrm{D}$ & Submitter talks too much & Wider happy/anticipatory face \\
\hline$:->$ & Hey, hey; smirk & \\
\hline \multirow[t]{2}{*}{$:-($} & Hoo hoo; disappointed & $\begin{array}{l}\text { "That comment makes me sad (or } \\
\text { mad)!" }\end{array}$ \\
\hline & & "Be prepared for trouble!" \\
\hline$:-<$ & Really sad & \\
\hline$:-\mathrm{C}$ & Really disappointed & \\
\hline$:-\mid$ & Hmmm; contemplation & Boring \\
\hline$:-\mathrm{O}$ & Uh oh! & \\
\hline$:-\mathrm{O}$ & Submitter is shocked. & Submitter is singing \\
\hline
\end{tabular}




\begin{tabular}{|c|c|c|}
\hline$\#-p$ & "Oh, nooooo!" (Remember & \\
\hline & Mr. Bill??) & \\
\hline$:-p$ & Nyah! Nyah! & Submitter is sticking tongue out \\
\hline |-P & Yuck! & \\
\hline$:-\}$ & Submitter has beard & Normal smiling face with pretty lips. \\
\hline \multirow[t]{3}{*}{$:-\{$} & Submitter has mustache. & \\
\hline & Submitter wear braces & Submitters lips are sealed. \\
\hline & & $\begin{array}{l}\text { Submitter has been punched in the } \\
\text { mouth! }\end{array}$ \\
\hline$:-) X$ & Submitter wears bow tie. & \\
\hline$:-Q$ & smoker & \\
\hline$<:-)$ & Dunce; dumb questions & \\
\hline $8-)$ & Submitter wears glasses & "I couldn t believe my eyes!" \\
\hline B-) & $\begin{array}{l}\text { Submitter wears horn-rimmed } \\
\text { glasses }\end{array}$ & A message from Batman \\
\hline $8-)$ & Submitter with glasses on forehead & Submitter is a little girl. \\
\hline ;-) & Wink & $\begin{array}{l}\text { "Take this message with a grain of } \\
\text { salt!" }\end{array}$ \\
\hline$>:-<$ & Submitter is mad. & \\
\hline :-@ & Submitter is screaming & \\
\hline$:-8($ & Condescending stare & \\
\hline$:-[$ & Biting criticism & \\
\hline $\mathrm{C}$ & Undecided & "That comment doesn't phase me." \\
\hline $\mid-]$ & Grimace & $\begin{array}{l}\text { "If I close my eyes tight, maybe it will } \\
\text { go away." }\end{array}$ \\
\hline$:-\mathrm{U}$ & Sarcasm & Speaking out of the side of one's mouth \\
\hline
\end{tabular}

